\title{
Characteristics of Rapid Rhythms Recorded Within Pulmonary Veins During Atrial Fibrillation
}

\author{
HIROSHI TADA, MEHMET ÖZAYDIN, HAKAN ORAL, BRADLEY P. KNIGHT, \\ AMAN CHUGH, CHRISTOPH SCHARF, FRANK PELOSI, \\ Jr., S. ADAM STRICKBERGER, and FRED MORADY \\ From the Division of Cardiology, Department of Internal Medicine University of Michigan, \\ Ann Arbor, Michigan
}

TADA, H., ET AL.: Characteristics of Rapid Rythms Recorded within Pulmonary Veins During Atrial Fibrillation. Rapid rhythms often arise in the pulmonary veins during atrial fibrillation (AF). The activation patterns within pulmonary veins during these rapid rhythms are not well described. In 39 patients with paroxysmal AF, ostial recordings were obtained during AF in 110 pulmonary veins with a decapolar distal ring catheter. Pulmonary vein tachycardia (PVT) was defined as a pulmonary vein (PV) rhythm that had a cycle length shorter than at the adjacent left atrium. During AF, PVT was recorded in $93 \%$ of PV's, usually in the form of intermittent bursts that had a mean duration of 1,325 $\pm 647 \mathrm{~ms}$ and mean cycle length of $125 \pm 20 \mathrm{~ms}$. The mean cycle length of continuous PVT's (141 \pm 25$)$ was longer than the mean cycle length of intermittent bursts of PVT $(P<0.05)$. The intermittent PVT's were associated with a shorter left atrial cycle length than were the continuous PVT's. In $90 \%$ of PVTs, complex activation patterns attributable to simultaneous recordings from two or more overlapping fascicles were present at a segment of the pulmonary vein ostium, and the mean cycle length of these recordings was $80 \pm 32 \mathrm{~ms}$. PVT during $A F$ is much more often intermittent than continuous. The relationship between PVT cycle length and left atrial cycle length suggests that PVT's influence the left atrium during AF. PVT must be distinguished from recordings within $P V$ 's that have a short cycle length as a result of simultaneous recordings from two or more overlapping fascicles. (PACE 2003; 26:1342-1347)

pulmonary vein, atrial fibrillation, tachycardia

\section{Introduction}

Several experimental and clinical studies have demonstrated the presence of rapid pulmonary vein rhythms that have a cycle length shorter than the cycle length recorded in the atrium during atrial fibrillation. ${ }^{1-12}$ These rapid rhythms may play a role in triggering and/or maintaining atrial fibrillation. ${ }^{2-5,7-12}$ The activation patterns recorded around the circumference of the ostium during rapid pulmonary vein rhythms in the setting of atrial fibrillation have not been described in detail. Therefore, the purpose of this study was to characterize the pulmonary venous ostial activation patterns during atrial fibrillation.

\section{Patient Characteristics}

\section{Methods}

The subjects of this study were 39 patients with drug-refractory, paroxysmal atrial fibrillation

\footnotetext{
Supported in part by a grant from the Gunma Prefecture Government and the Ellen and Robert Thompson Atrial Fibrillation Research Fund.

Address for reprints: Fred Morady, M.D., Division of Cardiology, B1F245, 1500 E. Medical Center Drive, Ann Arbor, MI 48109-0022. Fax: (734) 936-7026; e-mail:fmorady@umich.edu Received June 11, 2002; revised August 16, 2002; accepted October 3, 2002
}

who underwent segmental isolation of the pulmonary veins. ${ }^{13}$ There were 34 men and 5 women, and their mean age was $55 \pm 10$ years. The mean duration of symptomatic atrial fibrillation was $8.1 \pm 7.0$ years, and the patients experienced a mean of $12 \pm 10$ episodes/month. Four patients had hypertensive heart disease, and the remaining 35 patients had no structural heart disease. Echocardiography demonstrated a mean left ventricular ejection fraction of $0.59 \pm 0.10$ (range $0.40-$ 0.80 ), and a mean left atrial diameter of $40 \pm 5 \mathrm{~mm}$ (range 33-55 mm).

\section{Electrophysiologic Study Protocol}

Therapy with antiarrhythmic drugs was discontinued at least 48 hours before the procedure, and in the case of amiodarone, at least 6 weeks before the procedure. After informed consent was obtained, a 6 Fr or 7 Fr quadripolar electrode catheter (EP Technologies Inc., San Jose, CA, USA) was inserted into the right femoral vein and positioned in the distal coronary sinus. Transeptal catheterization was performed and a 7 Fr decapolar electrode catheter with $1 \mathrm{~mm}$ electrodes spaced at intervals of 4.5 or $6.0 \mathrm{~mm}$ in a distal ring configuration (Lasso, Biosense Webster, Inc., Diamond Bar, CA, USA) was inserted into the left atrium. A7 Fr quadripolar catheter with a 4-mm distal electrode and interelectrode spacing of 2-5-2 $\mathrm{mm}$ 
(EP Technologies Inc.) also was inserted into the left atrium for mapping and ablation. Heparin was administered as needed to maintain an activated clotting time of 250-350 seconds.

The surface leads and intracardiac electrograms were recorded digitally (EP Med Systems, Inc., Mount Arlington, NJ, USA). The filter settings were 30 to $500 \mathrm{~Hz}$ for bipolar electrograms and 0.05 to $200 \mathrm{~Hz}$ for unipolar electrograms. An indifferent electrode positioned on the right thigh was used for unipolar recordings. Pacing was performed from the coronary sinus with a stimulator (Model EP-3 Clinical Stimulator, EP Med Systems, Inc.) at twice the diastolic threshold and with a pulse width of $2 \mathrm{~ms}$.

\section{Study Protocol}

Pulmonary venous angiograms were performed to identify the location of the pulmonary vein ostia. Atrial fibrillation was present at the onset of the procedure in 19 patients, and was induced by rapid atrial pacing in the other 20 patients. The Lasso catheter was positioned sequentially within the left superior, left inferior, and right superior pulmonary veins. The Lasso catheter often could not be positioned within the right inferior pulmonary vein, and therefore electrograms recorded within this vein were not included in this study. The Lasso catheter was inserted 2-3 cm into the pulmonary vein and gradually withdrawn to within $5 \mathrm{~mm}$ of the ostium. During recordings of atrial fibrillation, the ablation catheter was positioned in the left atrium, adjacent to the pulmonary vein ostium in which the Lasso catheter was positioned.

Bipolar electrograms were recorded with the 10 pairs of adjacent electrodes of the Lasso catheter. When measuring the cycle length, only high frequency, discrete signals that had an amplitude $>0.2 \mathrm{mV}$ and that were separated by at least $50 \mathrm{~ms}$ were measured. Fractionated electrograms were defined as pulmonary vein potentials that had multiple deflections separated by $<50 \mathrm{~ms}$. When the cycle length inside the pulmonary vein was shorter than in the left atrium near that vein, this was considered evidence of a pulmonary vein tachycardia (Fig. 1). ${ }^{11}$ Pulmonary vein tachycardia was distinguished from shortening of the cycle length due to simultaneous recordings from two or more fascicles. This distinction was based on

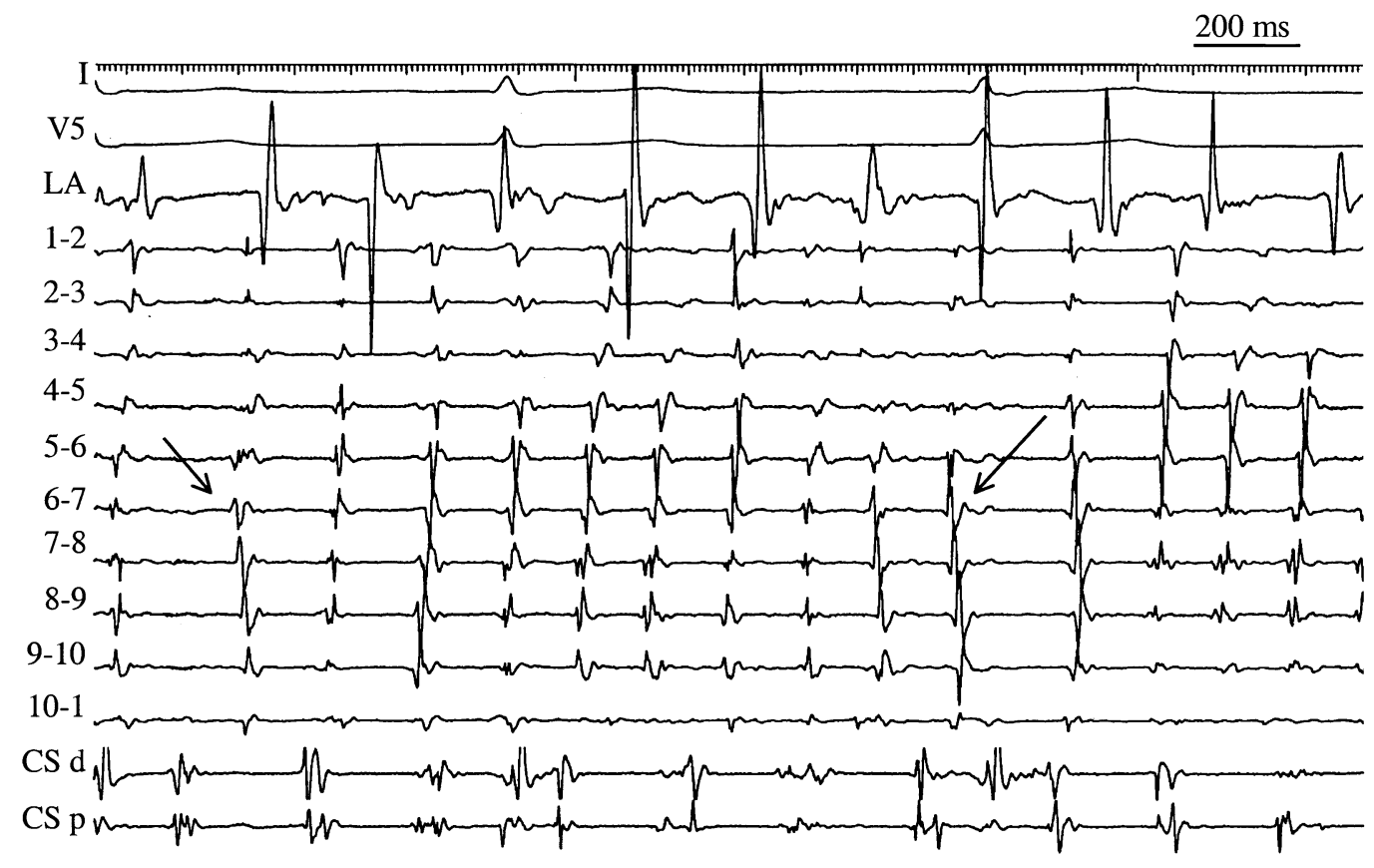

Figure 1. An example of an intermittent burst of tachycardia in the left superior pulmonary vein. Shown are leads $I$ and $V_{5}$, a bipolar electrogram recorded in the left atrium (LA) near the ostium of the left superior pulmonary vein, 10 bipolar electrograms recorded by a ring catheter positioned in the left superior pulmonary vein, and bipolar electrograms recorded in the distal (d) and proximal (p) coronary sinus (CS). Arrows mark the onset and offset of the tachycardia, which had a duration of 1,300 ms. The mean cycle length of the pulmonary vein tachycardia was $135 \mathrm{~ms}$, and the simultaneous mean cycle length in the left atrium adjacent to the vein was 190 ms. 
comparison of the activation pattern recorded by the electrodes that demonstrated the shortest cycle length with the activation patterns recorded by the adjacent electrodes of the ring catheter (Fig. 2). For the purposes of this study, a fascicle was defined as a discrete bundle of muscle fibers that generated a pulmonary vein potential, as validated by the disappearance of the pulmonary vein potential and electrical isolation of the fascicle in response to segmental ostial ablation. ${ }^{11,12}$

Electrograms that were suitable for analysis in this study were recorded in 35 left superior pulmonary veins, 35 left inferior pulmonary veins, 3 confluent left pulmonary veins, and 37 right superior pulmonary veins. The average duration of recordings in each vein was $3.1 \pm 1.4$ minutes.

\section{Statistical Analysis}

Continuous variables are expressed as mean \pm 1 standard deviation. Student's t test was used to compare 2 groups. Analysis of variance was used when comparisons involved $>2$ groups. For the purposes of analysis, the three left confluent pulmonary veins were categorized as left superior pulmonary veins. When group differences were found, one-way analysis of variance was followed by the Scheffe method to test the significance of the difference among means in all groups. Discrete variables were compared by Chi-square analysis. An overall Chi-square test for a $2 \pm \mathrm{n}$ table was constructed when comparisons involved $>2$ groups. A $\mathrm{P}$ value $<0.05$ was considered significant.

\section{Results \\ Prevalence of Pulmonary Vein Tachycardias}

A pulmonary vein tachycardia was recorded in 102 of 110 pulmonary veins (93\%). Pulmonary vein tachycardia was present during atrial fibrillation in 37 of 38 left superior pulmonary veins $(97 \%), 33$ of 37 right superior pulmonary veins $(89 \%)$, and 32 of 35 left inferior pulmonary veins $(91 \%)$. There was no significant difference in the prevalence of pulmonary vein tachycardia among the three pulmonary vein locations $(\mathrm{P}=0.4)$. The prevalence of pulmonary vein tachycardia was similar during spontaneous (50/52 veins, 96\%) and induced (52/58 veins, 90\%) atrial fibrillation $(\mathrm{P}=0.2)$.

\section{Intermittent vs Continuous Pulmonary Vein Tachycardia}

Eighty-seven percent of pulmonary vein tachycardias occurred as intermittent bursts that had a mean duration of $1,325 \pm 647 \mathrm{~ms}$ and a mean cycle length of $125 \pm 20$ ms (Fig. 1). Thirteen percent of pulmonary vein tachycardias were contin- uous during the entire recording interval, and had a mean cycle length of $141 \pm 25$ (Fig. 3). The mean cycle length of the intermittent pulmonary vein tachycardias was significantly shorter than the mean cycle length of the continuous pulmonary vein tachycardias $(\mathrm{P}<0.05)$. The activation pattern of intermittent pulmonary vein tachycardias was variable (Fig. 1), whereas the activation pattern during continuous pulmonary vein tachycardias was constant (Fig. 3).

The mean left atrial cycle length adjacent to the pulmonary vein was $173 \pm 40 \mathrm{~ms}$ during intermittent pulmonary vein tachycardias, and $199 \pm$ $36 \mathrm{~ms}$ during continuous pulmonary vein tachycardias. In both cases, the left atrial cycle lengths were significantly longer than the simultaneous pulmonary vein tachycardia cycle lengths $(\mathrm{P}<$ 0.001). The left atrial cycle length recorded during intermittent pulmonary vein tachycardias was significantly shorter than during continuous pulmonary vein tachycardias $(\mathrm{P}<0.05)$.

\section{Simultaneous Recordings from two or More Fascicles}

During $90 \%$ of pulmonary vein tachycardias, 1-3 of the 10 pairs of bipoles of the ring catheter demonstrated a shorter cycle length than the other bipoles, attributable to simultaneous recordings of depolarizations from 2 or more fascicles (Fig. 2). The mean cycle length in these recordings was $80 \pm 32 \mathrm{~ms}$, which was significantly shorter than the mean tachycardia cycle length of $125 \pm 20 \mathrm{~ms}$ in the adjacent recordings $(\mathrm{P}<0.001)$.

\section{Main Findings}

\section{Discussion}

In patients with paroxysmal atrial fibrillation, pulmonary vein tachycardias are present in approximately $90 \%$ of left superior, right superior, and left inferior pulmonary veins, regardless of whether the atrial fibrillation is spontaneous or induced by rapid atrial pacing. The pulmonary vein tachycardias occur approximately 5 times more often as intermittent bursts that last 1-2 seconds than continuously, and have a shorter mean cycle length when intermittent than when continuous. The association between pulmonary vein tachycardia cycle length and atrial fibrillation cycle length suggests that pulmonary vein tachycardias influence the left atrium during atrial fibrillation. Complex activation patterns caused by simultaneous recordings from two or more muscle fascicles are common during intermittent pulmonary vein tachycardias, and result in cycle lengths that are shorter than the actual tachycardia cycle lengths. 



Figure 2. A complex activation pattern recorded in a left superior pulmonary vein. Shown are lead II, 10 bipolar electrograms recorded by a Lasso catheter positioned in the left superior pulmonary vein, and bipolar electrograms recorded in the distal (d) and proximal ( $p$ ) coronary sinus (CS). (A) The rhythm recorded by electrodes 3-4 and 4-5 has a cycle length of $71 \mathrm{~ms}$, while the cycle length recorded by the other electrodes is approximately $150 \mathrm{~ms}$. The area within the dashed lines is shown in an expanded format in Figure 2B. (B) Comparison of the activation pattern recorded by electrodes 3-4 and 4-5 with the activation patterns recorded by the adjacent electrodes indicates simultaneous recordings from at least two overlapping fascicles. Simultaneous depolarizations in adjacent electrodes are encircled, demonstrating how the activation pattern recorded by electrodes 3-4 and 4-5 do not represent a single tachycardia, but a combination of depolarizations originating in overlapping fascicles. 


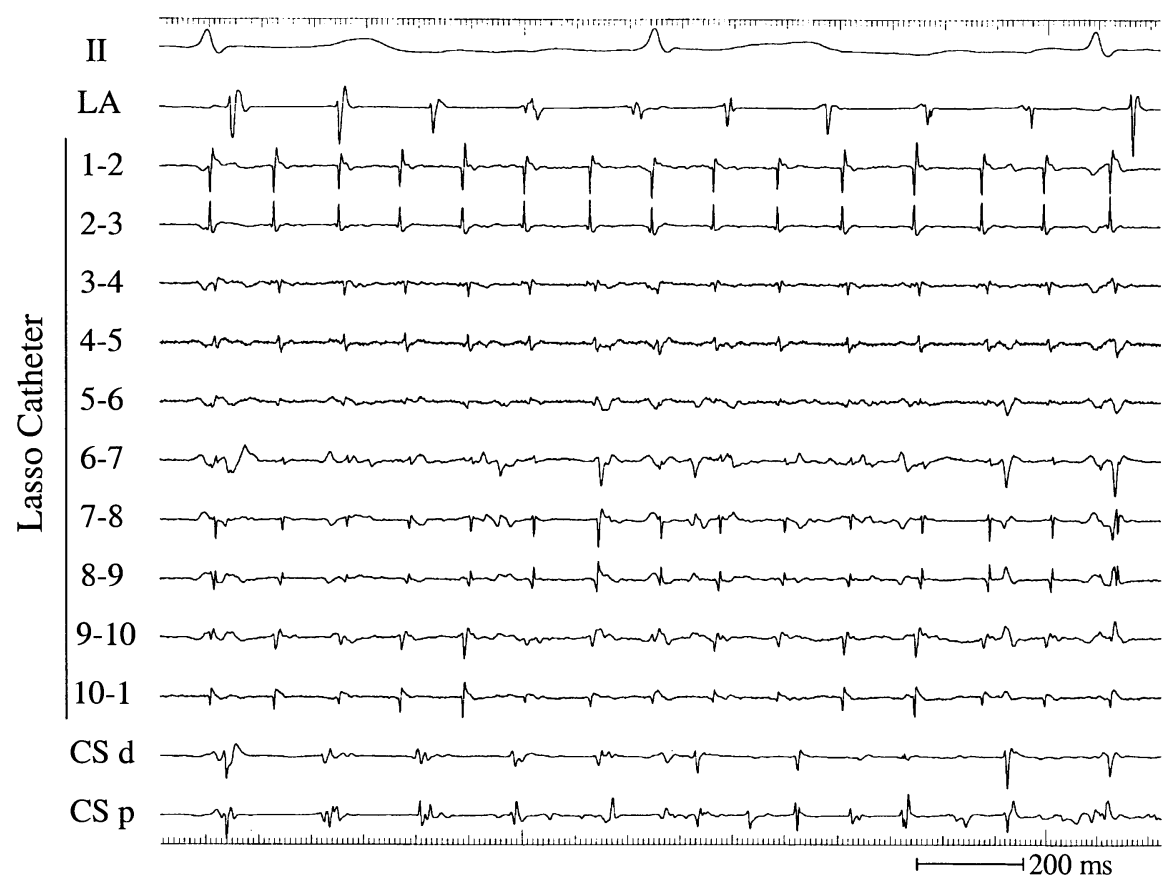

Figure 3. An example of a continuous tachycardia arising in a left inferior pulmonary vein. Shown are lead II, a bipolar electrogram recorded at the left atrium (LA) adjacent to the ostium of the left inferior pulmonary vein, 10 bipolar electrograms recorded by a Lasso catheter positioned in the left inferior pulmonary vein, and bipolar electrograms recorded in the distal (d) and proximal (p) coronary sinus (CS). The tachycardia had a cycle length of $124 \mathrm{~ms}$ and was present at all of the electrodes of the Lasso catheter during the entire 2 minutes of the recording. The simultaneous cycle length in the left atrium adjacent to the vein was $172 \mathrm{~ms}$.

\section{Prevalence of Pulmonary Vein Tachycardia}

In some prior studies, the prevalence of rapid pulmonary vein rhythms that had a cycle length shorter than in the left atrium during atrial fibrillation was reported to be only $12 \%-61 \%{ }^{6,8} \mathrm{In}$ these studies, pulmonary vein recordings were obtained with conventional catheters that had a linear array of electrodes. In contrast, with the ring catheter used in this study and in a prior study, ${ }^{11,12}$ the prevalence of pulmonary vein tachycardia was approximately $90 \%$. The tachycardias often were recorded at only some segments of the ostial circumference, and this is a likely explanation for why the prevalence of pulmonary vein tachycardia was underestimated in the prior studies that did not record electrograms from the entire circumference of the pulmonary vein. ${ }^{6,8}$

\section{Intermittent Pulmonary Vein Tachycardia}

Pulmonary vein tachycardias were found to be much more often intermittent than continuous. A prior experimental study of muscle fibers isolated from pulmonary veins of dogs prone to atrial fibrillation after several weeks of rapid atrial pacing, demonstrated a high prevalence of early afterde- polarizations. ${ }^{5}$ This suggests that triggered activity may be a mechanism of pulmonary vein tachycardias. High frequency, irregular rhythms that had a cycle length of $\leq 120 \mathrm{~ms}$ also were commonly found, ${ }^{5}$ consistent with the high prevalence of pulmonary vein tachycardia in the patients in this study. However, the reason that pulmonary vein tachycardias more often are intermittent than continuous remains to be determined.

\section{Relationship to Left Atrial Cycle Length}

Prior studies have presented evidence that pulmonary vein tachycardias may contribute to the maintenance of atrial fibrillation. ${ }^{2-5,7-12}$ In the present study, the cycle length of intermittent pulmonary vein tachycardias was found to be shorter than the cycle length of continuous pulmonary vein tachycardias, and the left atrial cycle length during atrial fibrillation was shorter during intermittent pulmonary vein tachycardias than during continuous pulmonary vein tachycardias. This observation provides evidence that pulmonary vein tachycardias influence the left atrial cycle length during atrial fibrillation, as would be expected if the pulmonary veins played a role in maintaining an episode of atrial fibrillation. 


\section{Recordings from Overlapping Fascicles}

The muscle sleeves that surround the pulmonary veins have a complex architecture, often with overlapping fascicles that are oriented in different directions. ${ }^{14,15}$ This complex arrangement of muscle fascicles explains why simultaneous recordings from more than one fascicle were commonly observed at some segments of the pulmonary vein ostia during episodes of atrial fibrillation.

Because simultaneous recordings from different fascicles by the same electrodes is a common phenomenon, the shortest cycle length recorded within a pulmonary vein often is not an accurate indicator of the true pulmonary vein tachycardia cycle length. This phenomenon can be recognized only by comparing the activation patterns recorded at adjacent sites along the ostia of the pulmonary veins. Therefore, if a conventional electrode catheter is used to record electrograms within the pulmonary veins, or if the activation patterns at adjacent electrodes of a ring catheter are not compared, it is likely that the cycle length of pulmonary vein tachycardia will erroneously be measured to be $\leq 40-50 \mathrm{~ms}$ shorter than the true tachycardia cycle length. This may account for why one study, in which conventional catheters were utilized, reported periodic clustering of cycle lengths between 40 and $120 \mathrm{~ms}$ within the pulmonary veins during atrial fibrillation, ${ }^{16}$ while in this and other studies, pulmonary vein tachycardias have been reported to have a mean cycle length of approximately $130 \mathrm{~ms} .^{11}$

\section{Limitations}

A limitation of this study is that the right inferior pulmonary vein often could not be cannu-

\section{References}

1. Cheung DW. Electrical activity of the pulmonary vein and its interaction with the right atrium in the guinea-pig. J Physiol 1981; 314:445-456.

2. Haissaguerre M, Jais P, Shah DC, et al. Spontaneous initiation of atrial fibrillation by ectopic beats originating in the pulmonary veins. N Engl J Med 1998; 339:659-666.

3. Chen SA, Hsieh MH, Tai CT, et al. Initiation of atrial fibrillation by ectopic beats originating from the pulmonary veins: Electrophysiological characteristics, pharmacological responses, and effects of radiofrequency ablation. Circulation 1999; 100:1879-1886.

4. Hwang C, Karagueuzian HS, Chen PS. Idiopathic paroxysmal atrial fibrillation induced by a focal discharge mechanism in the left superior pulmonary vein: Possible roles of the ligament of Marshall. J Cardiovasc Electrophysiol 1999; 10:636-648.

5. Chen YJ, Chen SA, Chang MS, et al. Arrhythmogenic activity of cardiac muscle in pulmonary veins of the dog: Implication for the genesis of atrial fibrillation. Cardiovasc Res 2000; 48:265-273.

6. Hsieh MH, Tai CT, Tsai CF, et al. Pulmonary vein electrogram characteristics in patients with focal sources of paroxysmal atrial fibrillation. J Cardiovasc Electrophysiol 2000; 11:953-959.

7. Kumagai K, Yasuda T, Tojo H, et al. Role of rapid focal activation in the maintenance of atrial fibrillation originating from the pulmonary veins. PACE 2000; 23:1823-1827.

8. Tse HF, Lau CP, Kou W, et al. Prevalence and significance of exit block during arrhythmias arising in pulmonary veins. J Cardiovasc Electrophysiol 2000; 11:379-386. lated with the ring catheter, and therefore data from this vein were not included in the study. A second limitation is that the study was purely descriptive, and the effects of radiofrequency ablation on the activation patterns recorded within the pulmonary veins were not evaluated. Another limitation is that the findings of the study do not rule out the possibility that complex activation patterns within pulmonary vein also may result from two or more microreentry circuits within a single muscle fascicle. Finally, because all of the patients in this study had paroxysmal atrial fibrillation, the results may not apply to chronic atrial fibrillation.

\section{Conclusions}

Rapid rhythms that have a shorter cycle length than the cycle length in the adjacent left atrium often are present during episodes of atrial fibrillation in patients with paroxysmal atrial fibrillation. These rapid rhythms may represent tachycardias that are generated within the pulmonary veins. The direct correlation between the pulmonary vein tachycardia cycle length and the left atrial cycle length suggests that these tachycardias influence the left atrium during atrial fibrillation. However, complex activation patterns that represent a combination of depolarizations generated by overlapping muscle fascicles and that have a shorter cycle length than the actual tachycardia cycle length are commonly recorded at segments of the pulmonary vein ostia. The failure to distinguish these complex activation patterns from pulmonary vein tachycardias generated by individual fascicles is likely to introduce significant errors in the measurement of the pulmonary vein tachycardia cycle length.

9. Wu TJ, Ong JJ, Chang CM, et al. Pulmonary veins and ligament of marshall as sources of rapid activations in a canine model of sustained atrial fibrillation. Circulation 2001; 103:11571163.

10. Chen PS, Wu T, Hwang C, et al. Thoracic veins and the mechanisms of non-paroxysmal atrial fibrillation. Cardiovasc Res 2002; 54:295301.

11. Oral H, Ozaydin M, Tada H, et al. Mechanistic significance of intermittent pulmonary vein tachycardia in patients with atrial fibrillation. J Cardiovasc Electrophysiol 2002; 13:645-650.

12. Oral H, Knight BP, Ozaydin M, et al. Segmental ostial ablation to isolate the pulmonary veins during atrial fibrillation. Feasibility and mechanistic insights. Circulation 2002; 106:1256-1262.

13. Oral H, Knight BP, Tada H, et al. Pulmonary vein isolation for paroxysmal and persistent atrial fibrillation. Circulation 2002; 105:10771081.

14. Saito T, Waki K, Becker A. Left atrial myocardial extension onto pulmonary veins in humans: Anatomic observations relevant for atrial arrhythmias. J Cardiovasc Electrophysiol 2000; 11:888-894.

15. Ho SY, Cabrera JA, Tran VH, et al. Architecture of the pulmonary veins: Relevance to radiofrequency ablation. Heart 2001; 86:265270.

16. O’Donnell D, Furniss SS, Bourke JP. Paroxysmal cycle length shortening in the pulmonary veins during atrial fibrillation correlates with arrhythmogenic triggering foci in sinus rhythm. J Cardiovasc Electrophysiol 2002; 13:124-128. 